\title{
What's your excuse for Foley use?
}

\section{Sarah L Krein, ${ }^{1,2}$ Sanjay Saint ${ }^{1,2}$}

${ }^{1}$ VA Ann Arbor Healthcare System, Center for Clinical Management Research, Ann Arbor, Michigan, USA ${ }^{2}$ Department of Internal Medicine, University of Michigan Medical School, Ann Arbor, Michigan, USA

\section{Correspondence to} Dr Sarah L Krein, VA Ann Arbor Healthcare System, Center for Clinical Management Research, P.O. Box 130170, Ann Arbor, MI 48113, USA;

skrein@umich.edu

Accepted 8 May 2015 Published Online First 1 June 2015

\section{CLinked}

- http://dx.doi.org/10.1136/ bmjqs-2015-004114

CrossMark

To cite: Krein SL, Saint $S$. BMJ Qual Saf 2015;24: 412-413.
He that is good for making excuses is seldom good for anything else.

\section{Benjamin Franklin}

Efforts to prevent catheter-associated urinary tract infection (CAUTI) are underway worldwide. ${ }^{1-4}$ Reducing indwelling urinary catheter (or 'Foley') use is a key component of most prevention initiatives, which makes sense given the evidence showing its effectiveness in reducing CAUTI rates. ${ }^{5}$ Such an approach, however, requires a specific focus on promoting the use of appropriate indications for a Foley at the time of insertion and throughout the duration of catheterisation. Unfortunately, data show substantial variability in both interpreting and applying such indications. ${ }^{6}$ The article by Murphy and colleagues ${ }^{7}$ uses robust qualitative methods to provide insights into decision-making about Foley insertion, including indications for use and the clinical context. This focus corresponds with what we and others have characterised as the socioadaptive aspects of CAUTI prevention, including behaviour change and unit culture, as compared with the technical components, such as urinary catheter reminders or stop orders. ${ }^{8}$ Murphy et al's work supports the use of certain strategies to promote more appropriate Foley use, such as better education about and availability of alternatives, including bladder scanning and external catheters (referred to as urinary sheaths in the paper). It also reinforces current wisdom about some of the presumed reasons why clinicians use Foley catheters, including issues related to workload and perceived convenience for clinicians (or patients) and other safety concerns (ie, pressure ulcer prevention). A multicenter study conducted in the USA focusing on CAUTI prevention practices similarly found that nursing convenience was perceived as a driving force underlying inappropriate urinary catheter use. ${ }^{9}$ As one of the nurses in that study stated: "convenience, unfortunately, is a high priority, and that is another factor, and especially with urinary catheters, yes, the workload will be increased if you have to take them to the bathroom or you have to change their bed a little more often..."9 Likewise, Harrod and colleagues $^{10}$ —also using qualitative methodsreported that hospital staff felt they had to choose between what they viewed as competing safety priorities (eg, effect of urinary catheter use on patients' risk of falls) when it comes to improvement initiatives to limit urinary catheter use. ${ }^{10}$

Other results reported by Murphy and colleagues highlight some emerging issues and approaches for addressing inappropriate or potential 'overuse' of Foleys. For example, the knee-jerk reaction illustrated by the emergency department physician who said "I really don't think about the decision at all" and the apparently unreflective favouring of Foley use to monitor urine output both suggest a need for better cognitive tools and strategies. This could include encouraging more mindful practice ${ }^{11}$ and better criteria and guidance for defining appropriate use, such as when hourly output monitoring might truly be required. ${ }^{12}$ On the other hand, concerns about avoiding contingencies and the use of combined weak indications and goals are more insidious and difficult to address, perhaps serving as fallback rationalisations or 'excuses' for actions rather than true decisions.

Among the several strengths of this paper is the in-depth nature of the findings and the focus on the insertion decision, highlighting specific attitudes or potential knowledge gaps that serve as points for intervention. Understanding how attitudes and perceptions related to Foley use differ across clinical settings (eg, emergency department vs medical ward) is also important in developing effective strategies to promote appropriate use. However, while generalisability in the statistical sense is not in question, the transferability of these findings to other hospitals and settings is of note. Specifically, at this hospital physicians were primarily responsible for urinary catheter insertion and this appeared to be 
a factor in decisions about catheter use for specific types of patients. Thus, how the findings related to patient age and gender might apply in hospitals where nursing staff have primary responsibility for Foley insertion-which is generally the case in most US hospitals-is an open question.

This limitation notwithstanding, this study reinforces our belief that it is the socio-adaptive aspects of CAUTI prevention that are often the greatest challenge for hospitals in their efforts to reduce catheter use and decrease CAUTI rates. ${ }^{13}$ While the work of Murphy and colleagues contributes further insight into clinician attitudes, perceptions, knowledge and behaviours related to Foley use, it also suggests areas for additional investigation, including better understanding of the patient perspective. While use of a Foley for patient comfort and dignity is often cited by clinicians, the reality is patients find indwelling catheters neither comfortable nor dignified. ${ }^{14}$

The study also indicates further work may be needed to improve clinician familiarity with existing alternatives or products, such as properly fitting external catheters and highly absorbent adult diapers, which for certain patients can be used to help ensure proper incontinence care without the use of an invasive device that heightens the risk for other complications. Another avenue for further study involves the clinical context and environmental factors that might promote or inhibit catheter use. Although some setting-specific interventions-such as in the emergency department or intensive care unit-have been developed, ${ }^{15}{ }^{16}$ more work in this domain is needed, including interventions that might target aspects of the built environment (eg, lack of restrooms and space constraints in a busy emergency department) as well as the prevailing culture and attitudes within a given area.

In sum, building on the work of Murphy and colleagues and with a bit more research and evidence the time has come, as the authors note, to 'challenge' some commonly held beliefs about Foleys. Indeed, rather than allowing the perpetuation of misguided beliefs and post hoc justification, we must be more proactive about promoting a more thoughtful and reasoned approach to indwelling urinary catheter use. As the quote that begins this editorial implies, Benjamin Franklin-credited with inventing the flexible catheter in 1752 when his brother suffered from bladder stones-disliked excuses. We feel the same way, especially when they are used to justify harmful practices such as putting in and keeping in unnecessary urinary catheters. So, what's your excuse for Foley use?

Disclaimer The views expressed in this article are those of the authors and do not necessarily reflect the position or policy of the Department of Veterans Affairs.

Competing interests None declared.

Provenance and peer review Not commissioned; internally peer reviewed.

\section{REFERENCES}

1 Fakih MG, George C, Edson BS, et al. Implementing a national program to reduce catheter-associated urinary tract infection: a quality improvement collaboration of state hospital associations, academic medical centers, professional societies, and governmental agencies. Infect Control Hosp Epidemiol 2013;34:1048-54.

2 Rosenthal VD, Todi SK, Alvarez-Moreno C, et al. Impact of a multidimensional infection control strategy on catheter-associated urinary tract infection rates in the adult intensive care units of 15 developing countries: findings of the International Nosocomial Infection Control Consortium (INICC). Infection 2012;40:517-26.

3 Apisarnthanarak A, Thongphubeth K, Sirinvaravong S, et al . Effectiveness of multifaceted hospitalwide quality improvement programs featuring an intervention to remove unnecessary urinary catheters at a tertiary care center in Thailand. Infect Control Hosp Epidemiol 2007;28:791-8.

4 Stephan F, Sax H, Wachsmuth M, et al. Reduction of urinary tract infection and antibiotic use after surgery: a controlled, prospective, before-after intervention study. Clin Infect Dis 2006;42:1544-51.

5 Meddings J, Rogers MA, Krein SL, et al. Reducing unnecessary urinary catheter use and other strategies to prevent catheter-associated urinary tract infection: an integrative review. BMJ Qual Saf 2014;23:277-89.

6 Greene MT, Fakih MG, Fowler KE, et al. Regional variation in urinary catheter use and catheter-associated urinary tract infection: results from a national collaborative. Infect Control Hosp Epidemiol 2014;35(Suppl 3):S99-106.

7 Murphy C, Prieto J, Fader M. "It's easier to stick a tube in": a qualitative study to understand clinicians' individual decisions to place urinary catheters in acute medical care. BMJ Qual Saf 2015;24:444-50.

8 Saint S, Howell JD, Krein SL. Implementation science: how to jump-start infection prevention. Infect Control Hosp Epidemiol 2010;31(Suppl 1):S14-17.

9 Saint S, Kowalski CP, Forman J, et al. A multicenter qualitative study on preventing hospital-acquired urinary tract infection in US hospitals. Infect Control Hosp Epidemiol 2008;29:333-41.

10 Harrod M, Kowalski CP, Saint S, et al. Variations in risk perceptions: a qualitative study of why unnecessary urinary catheter use continues to be problematic. BMC Health Serv Res 2013;13:151.

11 Kiyoshi-Teo H, Krein SL, Saint S. Applying mindful evidence-based practice at the bedside: using catheter-associated urinary tract infection as a model. Infect Control Hosp Epidemiol 2013;34:1099-101.

12 Meddings J, Saint S, Fowler KE, et al. The Ann Arbor criteria for appropriate urinary catheter use in hospitalized medical patients: results using the RAND/UCLA appropriateness method. Ann Intern Med 2015;162(9 Suppl):S1-34.

13 Saint S, Krein SL, Stock RW. Preventing hospital infections: real-world problems, realistic solution. New York, NY: Oxford University Press, 2015.

14 Saint S, Lipsky BA, Goold SD. Indwelling urinary catheters: a one-point restraint? Ann Intern Med 2002;137:125-7.

15 Fakih MG, Pena ME, Shemes S, et al. Effect of establishing guidelines on appropriate urinary catheter placement. Acad Emerg Med 2010;17:337-40.

16 Gokula RM, Smith MA, Hickner J. Emergency room staff education and use of a urinary catheter indication sheet improves appropriate use of foley catheters. Am J Infect Control 2007;35:589-93. 